\title{
Nuclear Molecular Gas in the Virgo Cluster S0 Galaxy NGC 4710
}

\author{
J.M. WROBEL \\ NRAO, P.O. Box O, Socorro, NM 87801, USA \\ and \\ J.D.P. KENNEY \\ Dept. of Astronomy, Box 6666, Yale University, New Haven, CT 06511, USA
}

\begin{abstract}
The $\mathrm{CO}(\mathrm{J}=1 \rightarrow 0)$ emission from NGC 4710, a star-forming So galaxy in the Virgo Cluster, was synthesized with spatial and velocity resolutions of $7^{\prime \prime}$ and $26 \mathrm{~km} \mathrm{~s}^{-1}$, respectively. The CO shows a compact morphology and co-rotates with the galaxy's stars and nuclear optical emission line gas. Analysis of the $\mathrm{CO}$ distribution and kinematics indicates that the nuclear molecular gas is probably gravitationally unstable, and this may explain why the galaxy is presently forming stars. Four possible origins for the nuclear molecular gas are considered. An origin via bulge star ejecta being deposited into a residual interstellar medium is favored.

This work is fully described in the 1992 November 1 issue of The Astrophysical Journal. NRAO is operated by Associated Universities, Inc., under cooperative agreement with the National Science Foundation.
\end{abstract}

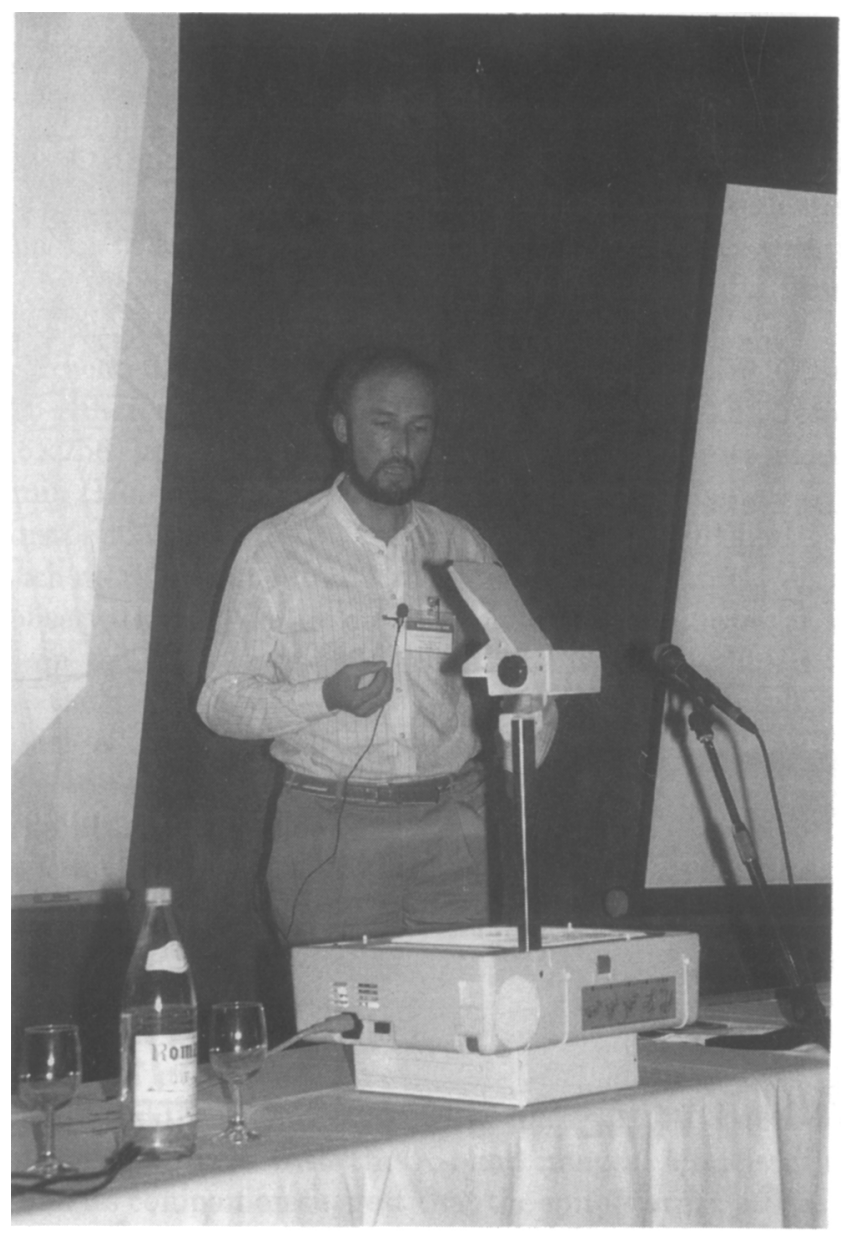

D. Pfenniger 


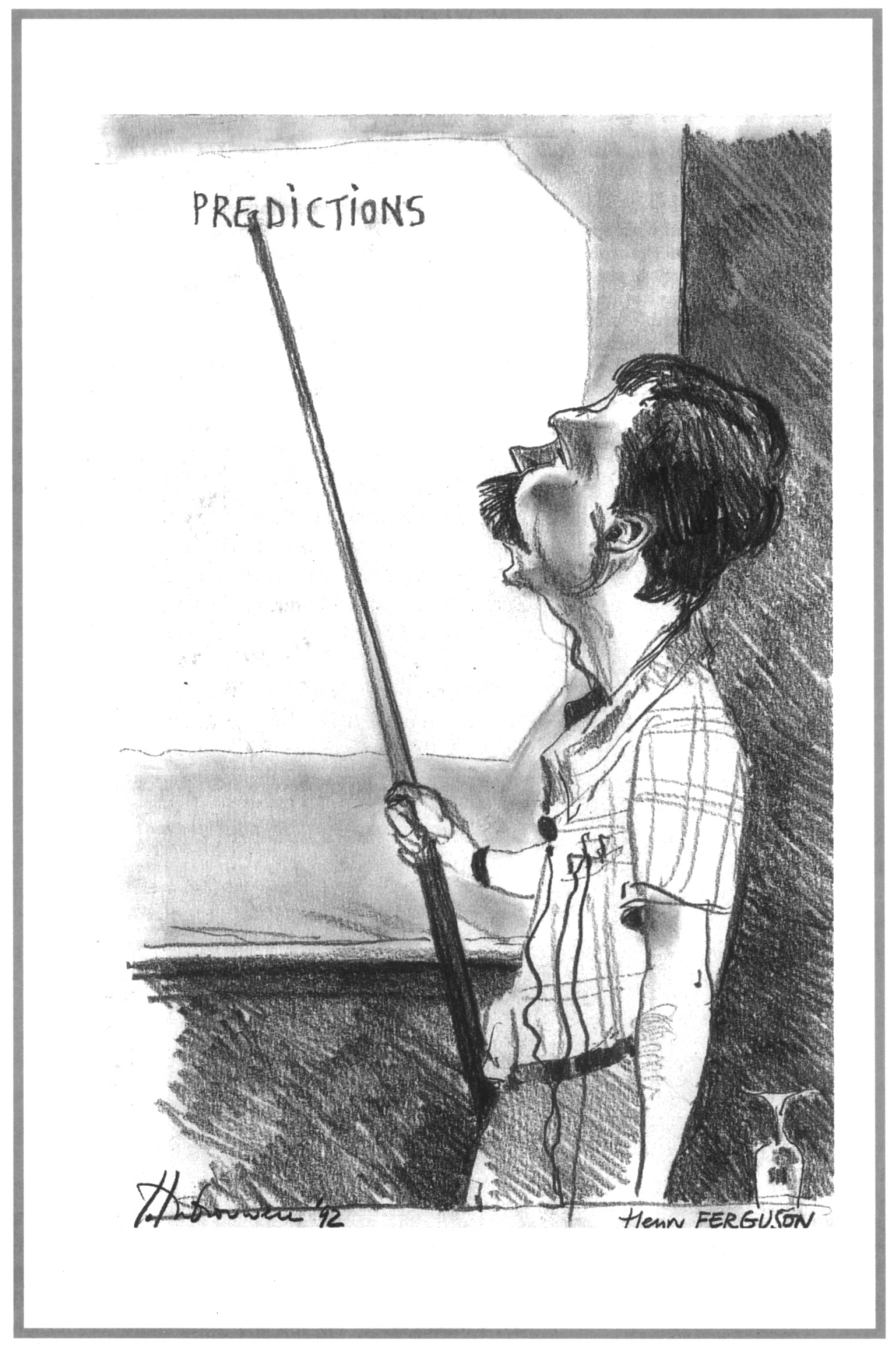

